\title{
A Moxa Stimulation on the Leg Affected the Function of Stomach via Autonomic Nerve System and Polymodal Receptors
}

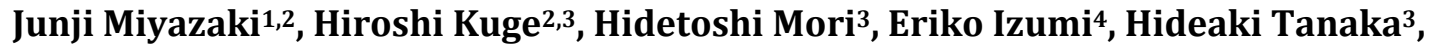 \\ Mayumi Watanabe ${ }^{3,5^{*}}$ \\ ${ }^{1}$ Department of Acupuncture, Faculty of Health Care Sciences, Takarazuka University of Medical and Health \\ Care, Takarazuka, Japan \\ ${ }^{2}$ Department of Anesthesiology, Osaka Medical College Hospital, Osaka, Japan \\ ${ }^{3}$ Department of Health, Faculty of Health Science, Tsukuba University of Technology, Tsukuba, Japan \\ ${ }^{4}$ Department of a Practitioner of Acupuncture and Moxibustion, Heisei College of Medical Technology, Osaka, \\ Japan \\ ${ }^{5}$ Department of Medical Informatics, Niigata University Medical and Dental Hospital, Niigata, Japan \\ Email: *watanabem62@gmail.com
}

Received 30 January 2016; accepted 28 May 2016; published 31 May 2016

Copyright (C) 2016 by authors and Scientific Research Publishing Inc.

This work is licensed under the Creative Commons Attribution International License (CC BY).

http://creativecommons.org/licenses/by/4.0/

c) (i) Open Access

\section{Abstract}

The moxa therapy is a popular traditional hyperthermia therapy in East Asia. The moxa is made from dried mugwort (Artemisiaargyi). The moxa is usually put on a meridian point and then is burnt on to obtain a hyperthermic stimulation to the skin. However, very few researches have been studied in the effects and mechanism of the moxa therapy. Therefore, in this study, we gave the moxa stimulation to healthy subjects. In the moxa group, the moxa was lit on the ST36 (Zusanli: an acupuncture point in anterior tibialis of the leg) for 5 minutes. Their responses were traced with electrogastrogram (EGG) and assessed the change of gastric function. At the same time, their heart rate (HR) was recorded and observed to study the mechanism of moxa treatment. And then their results were compared with those of the control group. After the moxa stimulation, EGG showed significant increase and stayed $3.0-3.1 \mathrm{cpm}$ while HR prominently decreased. On the other hand, the control group did not indicate such results. Those results are common to the response of acupuncture-like stimulation by the reflex arc which consists of receptor, sensory neuron, central nervous system, motor neuron and effector. It may be explained as below: the elements of the acupuncture-like stimulation and the moxa are pain and hyperthermia respectively and they both are received by polymodal receptors. Thus the moxa stimulation applied on ST36 may be effective to enhance stomach function.

"Corresponding author.

How to cite this paper: Miyazaki, J., Kuge, H., Mori, H., Izumi, E., Tanaka, H. and Watanabe, M. (2016) A Moxa Stimulation on the Leg Affected the Function of Stomach via Autonomic Nerve System and Polymodal Receptors. Health, 8, 749-755.

http://dx.doi.org/10.4236/health.2016.88078 
Keywords

Moxa Therapy, Electrogastrogram (EGG), Heart Rate (HR), Polymodal Receptor (PMR)

\section{Introduction}

Oriental medicine is a broad range of medicine practices sharing common concepts which have been developed in China and are based on a tradition of more than 2500 years, including such as the acupuncture therapy, the moxa therapy and the massage therapy. Nowadays it is widely used in China and it is also used in the West [1]. In 1997, the U.S. National Institutes of Health published a Consensus Statement on the use and effectiveness of the acupuncture therapy for a variety of conditions. In 2003, World Health Organization reported that acute epigastralgia (in peptic ulcer, acute and chronic gastritis, and gastrospasm) was diseases, symptoms or conditions for which acupuncture had been proved-through controlled trials- to be an effective treatment [2]. However, there is room for further research into oriental medicine.

In studies of oriental medicine, electrogastrogram (EGG) is often used. EGG can non-invasively assess the change of gastric activity. Therefore, it is often used in study in dysfunction of the digestive tract. In addition, heart rate (HR) which indicates the status of autonomic nervous system is often studied to assess the effects of oriental medicine.

Walter C. Alvarez is an early pioneer of EGG and he reports that the electrical activity of the normal human consists in regular pacesetter potential (frequency $0.05 \mathrm{~Hz}=3.0 \mathrm{cpm}$ ) [3] [4]. EGG records the electrical signals that travel through the stomach muscles' constriction; therefore, it can detect the wave-like contractions of the stomach (peristalsis).

Along the way, many researchers investigate effects of oriental medicine, especially those of acupuncture-like stimulation, however, very few has investigated moxa stimulation though moxibustion as well as acupuncture (or acupuncture-like stimulation) on bedside [5]-[9]. The moxa therapy is a traditional and popular therapy using moxa made from dried mugwort (Artemisiaargyi). Suppliers usually age the mugwort and grind it up to a fluff; practitioners burn the fluff or process it further into a cigar-shaped stick or a rigid card board tube. They can use it indirectly, with acupuncture needles, or burn it on the patients' skin (but they do not get burnt). Therefore, in this study, we give moxa stimulation to healthy subjects on their ST36 (an acupuncture point on the leg, which is called Zusanli). And then we assess their responses with EGG and HR to study the mechanism of moxa treatment.

\section{Materials and Methods}

\subsection{Subjects}

First, Healthy volunteers $(\mathrm{n}=15$; male $=7$, female $=8$; average $39.0 \pm 7.1$ years old $)$ were divided into two groups, the control group $(\mathrm{n}=8$; male $=7$, female $=8 ; 40.4 \pm 6.6)$ and the moxibustion (moxa) group $(\mathrm{n}=7$; male $=7$, female $=8 ; 37.5 \pm 7.7$ ) using the envelope allocation method (Figure 1). The experiments were conducted in a room with the temperature set at $25.0^{\circ} \mathrm{C} \pm 0.5^{\circ} \mathrm{C}$ and $50.0 \% \pm 5.0 \%$ humidity throughout the experimental sessions.

This study adhered strictly to the principles of the Declaration of Helsinki. Before the study began, the participants were told about the purpose and procedures of the study, and all participants signed consent forms. This study was approved by the institutional review board of Takarazuka University of Medical and Health Care.

\subsection{Stimulation}

\subsubsection{Moxibustion (Moxa) Stimulation}

For the moxa stimulation, the Kamaya mini moxa (Kamaya Mogusa Co., Ltd, Tokyo, Japan), a kind of indirect moxa, was used. This moxa is a rigid cardboard tube containing a plug of moxa and it was pushed partly out of the tube (Figure 2(a)).

In both groups, subjects took rest in the supine position for 30 minutes with the moxa, which was vertically placed on left-leg Zusanli (ST36) with the adhesive at the base of the tube. And then, in the moxa group it was 


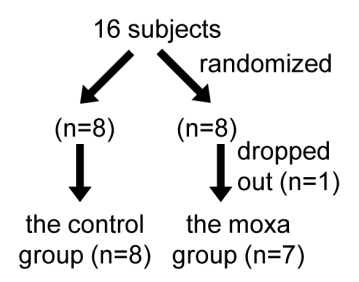

Figure 1. Healthy volunteers $(n=15$; male $=7$, female $=8$ ) were divided into two groups, the control group $(\mathrm{n}=8$; male $=7$, female $=8$ ) and the moxa group $(n=7$; male $=7$, female $=8$ ) using the envelope allocation method.

(a)

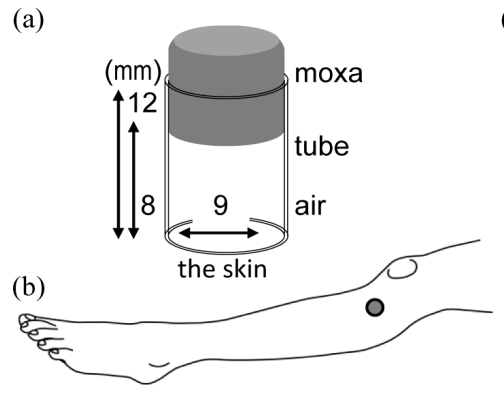

(c)

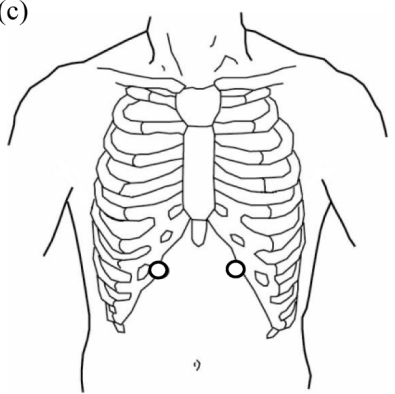

Figure 2. (a)The moxa is a rigid cardboard tube containing a plug of moxa which was pushed partly out of the tube. (b) The moxa was put onZusanli ( $\bullet$ : ST36) which is located on the tibialis anterior muscle. (c) Kinetic changes in electrogastrogram were recorded with two disposable electrodes which were put right and left Burong (०: ST19).

lit for five minutes of the moxa period. In the control group it was not lit but kept the same position for five minutes of the no-moxa stimulation period. In both groups, it was removed after the five minutes.

Their last food intake was more than two hours before this experiment. The intensity of stimulation was monitored by Visual Analogue Scale (VAS) to prevent causing burn injury or discomfort to the subjects.

\subsubsection{ST36 (Zusanli)}

ST36 is located on the tibialis anterior muscle [2] [10] (Figure 2(b)). ST36 belongs to the stomach meridian and it have been known to help stomach function such as preventing stomach pains or vomiting as described in the “Huangdi Neijing” (Yellow Emperor's classic of internal medicine) of the Western Han dynasty about 2 B.C.

\subsection{Measurement}

\subsubsection{The Skin Temperature on ST36 (Zusanli) on Left-Leg}

To obtain the skin temperature on ST36, type K thermocouple (ANBE SMT co., Yokohama, Japan), TC-08 Thermocouple Data Logger (Pico Technology Limited, Cambridgeshire, UK) and Pico log recorder (Pico Technology Limited, Cambridgeshire, UK) were used.

\subsubsection{Electrogastrogram (EGG)}

In accordance with earlier studies, kinetic changes in EGG (EG, Nipro Corporation, Osaka, Japan) were recorded every 256 seconds [11] [12]. Two disposable electrodes (3M ${ }^{\mathrm{TM}} \operatorname{Red~Dot~}^{\mathrm{TM}}$; 3M Japan Limited, Tokyo, Japan) were put right (criterion) and left (measurement) ST19 (Burong) as shown in Figure 2(c). And then thus obtained data was analyzed by a dedicated software, EGS2 (Gram Corporation, Saitama, Japan). EGG data were shown in 9 points: before the moxa stimulation, during the stimulation (moxa), after 4, 6, 10, 14, 18, 22 and 26 after the moxa stimulation.

\subsubsection{ST19 (Burong) as Measurement Point of EGG}

On the upper abdomen, 6 B-cun superior to the centre of the umbilicus, 3 B-cun lateral to the anterior meridian 
line. It also belongs to the stomach meridian and it is one of the nearest acupuncture points to the stomach organ among the stomach meridian acupuncture points [3] (Figure 2(c)).

\subsubsection{Heart Rate (HR) Measurement}

To assess the HR, Heart Rhythm Scanner (Ver. 2.0) and Heart Rate Variability Analysis System (Biocom Technologies Inc., WA, USA) was used [13] [14]. The data of HR were recorded as below: before the moxa stimulation, during the moxa stimulation (moxa), immediately after the moxa stimulation and then, it was traced for every 5 minutes afterward.

\subsection{Statistical Analysis}

Changes of the data (the skin temperature, EGG and HR) of both groups were analyzed using one-way ANOVA and Fisher's LSD multiple comparison test. SPSS Advanced Models version 15 was used as the statistical analysis software. The level of significance was set at $\mathrm{p}<0.05$. The values presented were mean $\pm \mathrm{SE}$.

\section{Results}

\subsection{The Skin Temperature with the Moxa Stimulation}

In the moxa group, the skin temperature rose toward a peak $\left(46.3^{\circ} \mathrm{C} \pm 2.5^{\circ} \mathrm{C}\right)$ until one minute after the removal of the moxa (Figure 3). In the control group, such a change was not found. Both groups were analyzed using one-way ANOVA and Fisher's LSD multiple comparison test.

\subsection{After the Moxa Stimulation EGG Showed Significant Increase and Stayed 3.0 - $3.1 \mathrm{cpm}$}

In the control group, compared to the before the experiment (3.6 $\pm 0.4 \mathrm{cpm}$ ), EGG showed significant decrease after 10 (2.5 $\pm 0.5 \mathrm{cpm}, \mathrm{p}=0.038), 14$ ( $2.4 \pm 0.2 \mathrm{cpm}, \mathrm{p}=0.019), 18$ (2.4 $\pm 0.2 \mathrm{cpm}, \mathrm{p}=0.022)$, and $22(2.3 \pm$ $0.3 \mathrm{cpm}, \mathrm{p}=0.015$ ) minutes afterwards. On the other hand, the moxa group, compared to before the stimulation $(2.3 \pm 0.3 \mathrm{cpm})$, EGG showed significant increase and stayed $3.0-3.1 \mathrm{cpm}$ as below: 18 (3.1 $\pm 0.1 \mathrm{cpm}, \mathrm{p}=$ $0.010), 22$ ( $3.0 \pm 0.1 \mathrm{cpm}, \mathrm{p}=0.023$ ) and $26(3.1 \pm 0.2 \mathrm{cpm}, \mathrm{p}=0.010)$ minutes after the stimulation (Figure 4). Both groups were analyzed using one-way ANOVA and Fisher's LSD multiple comparison test.

\subsection{The Heart Rate (HR) Significantly Decreased after the Moxa Stimulation.}

In the moxa group, HR significantly decreased immediately after the stimulation $(62.6 \pm 3.2 / \mathrm{min}, \mathrm{p}=0.030), 5$ (62.1 $\pm 3.1 / \mathrm{min}, \mathrm{p}=0.011), 10(61.6 \pm 3.3 / \mathrm{min}, \mathrm{p}=0.005)$ and $15(60.9 \pm 3.1 / \mathrm{min}, \mathrm{p}=0.001)$ minutes after the

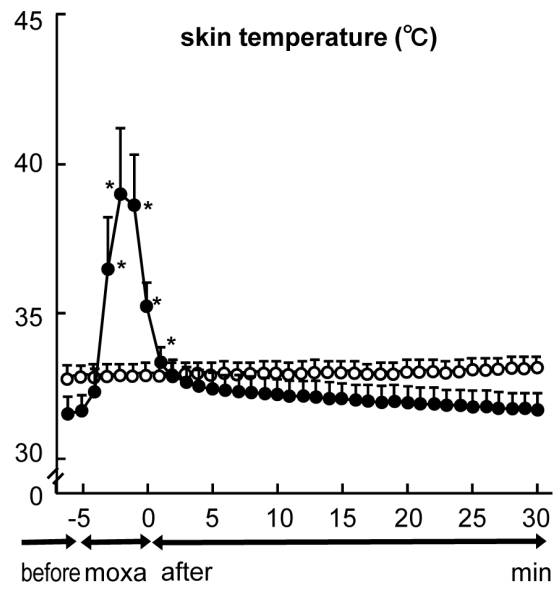

Figure 3. The kinetic skin temperature: In the moxa group, the skin temperature rose toward a peak $\left(46.3^{\circ} \mathrm{C} \pm 2.5^{\circ} \mathrm{C}\right)$ until one minute after the removal of the moxa tube. In the control group, such a change was not found. $\circ$ : the control group, $\bullet$ : the moxa group, $\mathrm{p}<0.05$. 
moxa stimulation compared to that of before the stimulation $(65.7 \pm 3.6 / \mathrm{min})$. On the other hand, such difference was not found in the control group (Figure 5). Both groups were analyzed using one-way ANOVA and Fisher's LSD multiple comparison test.

\section{Discussion}

\subsection{The Moxa Stimulation Increased Gastric Potential.}

In this study, we applied the moxa, another kind of somatosensory stimulation, applied to ST36 in anterior tibialis of the leg and recorded its effects with EGG. Our results indicated that the moxa group increased and maintained the value of EGG at the level of $3.0-3.1 \mathrm{cpm}$ (Figure 4). It is reported that the electrical activity of the normal human consists in regular pacesetter potential (frequency $0.05 \mathrm{~Hz}=3.0 \mathrm{cpm}$ ) [4] and that amplitude and velocity of this activity increased as it approach the pylorus [15]. Thus the moxa stimulation increased gastric potential as well as acupuncture-like stimulation did. Therefore, the moxa stimulation may be effective to enhance stomach function such as peristalsis.

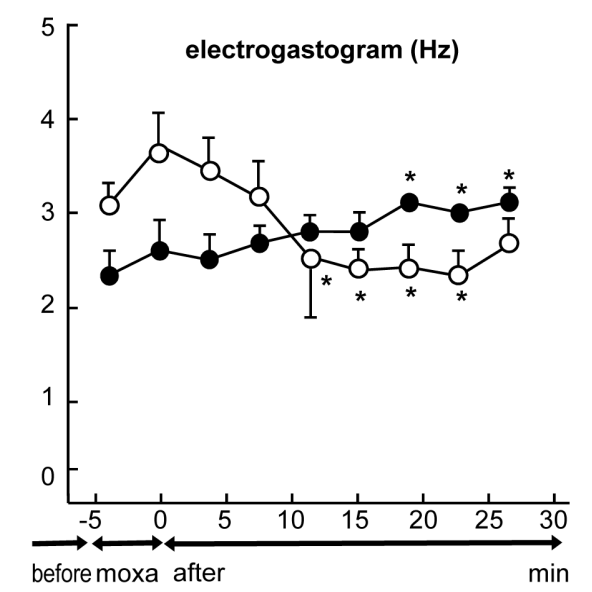

Figure 4. The kinetic change of electrogastrogram (EGG): In the control group, compared to before the experiment EGG showed significant decrease at four points after 10 minutes after the stimulation. On the other hand, the moxa group, compared to before the experiment, EGG showed significant increase and stayed 3.0 - $3.1 \mathrm{cpm}$. ०: the control group, $\bullet$ : the moxa group, $\mathrm{p}<0.05$.

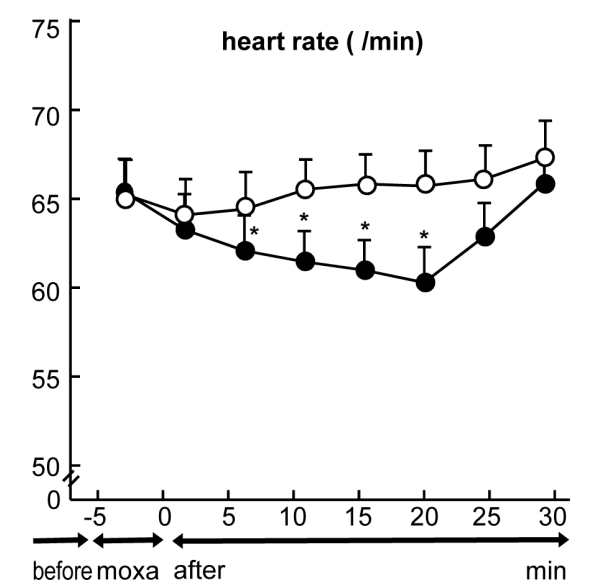

Figure 5. In the moxa group, HR significantly showed decrease from 0 to 20 minutes after the moxa stimulation. On the other hand, such difference was not found in the control group. $\odot$ : the control group, $\bullet$ the moxa group, $\mathrm{p}<0.05$. 


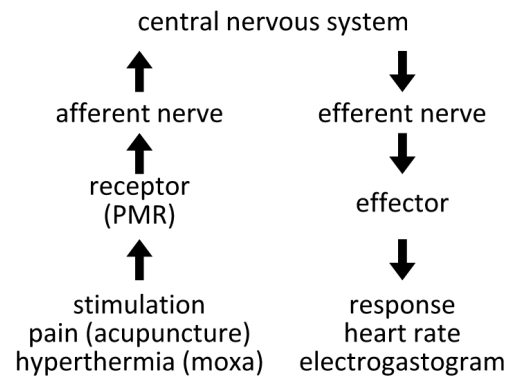

Figure 6. The reflex arc consists of receptor, afferent nerve, central nervous system, efferent nerve and effector.

\subsection{The Moxa Stimulation Decreased HR via Autonomic Nerve System and Polymodal Receptor (RPM)s}

Those results are common to the response of acupuncture-like stimulation by the reflex arc which consists of receptor, afferent nerve, central nervous system, efferent nerve and effector. It may be explained as below: the element of the acupuncture-like stimulation and the moxa are pain and hyperthermia respectively and they both are received by polymodal receptors (Figure 6).

Our study indicated that HR decreased after the moxa stimulation on ST36 (Figure 5). Researchers reported that acupuncture-like stimulation to the leg decreased HR via polymodal receptors (PMR)s [15]-[19]. PMRs are characterized by activation from a wide variety of stimulations since the skin is exposed to various forms of stimulations from the external environment and they are widely distributed over the skin as well as in the somatic and visceral deep tissues.

As all subjects in the moxa group undoubtedly received thermal stimulation (maximum temperature; $45^{\circ} \mathrm{C}$ ) (Figure 3), it is understood that PMRs were activated as well as acupuncture-like stimulation. Therefore, HR decreased after the moxa stimulation. In short, it may be considered that the moxa stimulation could induce similar effect as acupuncture-like stimulation via autonomic nerve system and RPMs.

\subsection{The Moxa Stimulation May be Effective for Regulating Gastric Function}

It is reported that the acupuncture-like stimulation (electrical acupuncture) to ST36 enhanced the regularity of gastric activity and that may be an option for treatment of gastric dysrhythmia or non-ulcer dyspepsia [20] [21]. Therefore, acupuncture may enhance the regularity of gastric activity in diabetic patients [6].

In this study, to investigate the effects of the moxa treatment we used EGG, which records the gastric change. Therefore, ST36 and ST19 were selected as points for the moxa stimulation and recording EGG respectively.

However, our study has several limitations. First, the number of subjects were limited in this study, therefore, further research, such as larger scale studies, are required to support the findings. Second, we did not stimulate other area. The specific nature of ST36 (the point of the moxa stimulation) and ST19 (the observation point of EGG) was not negligible because both points belong to stomach meridians since it has been said that they are intimately related to the stomach condition [21]. These would be valuable factors to assess in any future study.

\section{Conclusion}

The moxa stimulation on ST 36 may be effective to enhance stomach function, and it decreases HR via RPMs and autonomic nerve system. Therefore it may be effective for regulating gastric function.

\section{References}

[1] National Institude of Health (1997) Acupuncture. NIH Consensus Statement, 15, 1-34.

[2] World Health Organization (2002) Acupuncture: Review and Analysis of Reports on Controlled Clinical Trials. World Health Organization, Geneva.

[3] Alvarez, W.C. (1922) The Electrogastrogram and What Is Shows. Journal of the American Medical Association, 78, 1116-1119. http://dx.doi.org/10.1001/jama.1922.02640680020008 
[4] Counturier, D., Rose, C., Paologgi, J. and Debray, C. (1972) Electrical Activity of the Normal Human Stomach, A Compareative Study of Recorings Obtained from Serosal and Mucosal Side. The American Journal of Digestive Diseases, 17, 969-976.

[5] Yin, J. and Chen, J.D. (2010) Gastrointestinal Motility Disorders and Acupuncture. Autonomic Neuroscience, 157, 3137. http://dx.doi.org/10.1016/j.autneu.2010.03.007

[6] Chang, C.S., Ko, C.W., Wu, C.Y. and Chen, G.H. (2001) Effect of Electrical Stimulation on Acupuncture Points in Diabetic Patients with Gastric Dysrhythmia: A Pilot Study. Digestion, 64, 184-190. http://dx.doi.org/10.1159/000048860

[7] Chang, C.S., Chou, J.W., Wu, C.Y., Chang, Y.H., Ko, C.W. and Chen, G.H. (2002) Atropine-Induced Gastric Dysrhythmia Is Not Normalized by Electroacupuncture. Digestive Diseases and Sciences, 47, 2466-2472. http://dx.doi.org/10.1023/A:1020599707395

[8] Guo, L.K., Zhang, C.X. and Guo, X.F. (2011) Long-Term Efficacy and Safety Research on Functional Dyspepsia Treated with Electroacupuncture and Zhizhu Kuanzhong Capsule. Chinese Acupuncture \& Moxibustion, 31, 10711077.

[9] Noguchi, E. (2010) Acupuncture Regulates Gut Motility and Secretion via Nerve Reflexes. Autonomic Neuroscience, 156, 15-18. http://dx.doi.org/10.1016/j.autneu.2010.06.010

[10] World Health Organization (2008) WHO Standard Acupuncture Point Locations in the Western Pacific Region. WHO Western Pacific Region.

[11] Shiomi, M., Imai, K. and Sakita, M. (2003) Effect of Acupuncture Stimulation on Optokinetic Motion Sickness Monitored by Electrogastrograms. JSAM, 53, 71-80.

[12] Japan Society of Neurovegetative Research Volume. (2007) Autonomic Nervous Function Test. 4th Edition, Bunkodo Co., Ltd, Tokyo, 313-315.

[13] Bolanos, M., Nazeran, H. and Haltiwanger, E. (2006) Comparison of Heart Rate Variability Signal Features Derived from Electrocardiography and Photoplethysmography in Healthy Individuals. In: Engineering in Medicine and Biology Society. EMBS’06 28th Annual International Conference of the IEEE, New York, 30 August-3 September 2006, 42894294. http://dx.doi.org/10.1109/iembs.2006.260607

[14] Russoniello, C.V., Pougtachev, V., Zhirnov, E. and Mahar, M.T. (2010) A Measurement of Electrocardiography and Photoplethesmography in Obese Children. Applied psychophysiology and biofeedback, 35, 257-259. http://dx.doi.org/10.1007/s10484-010-9136-8

[15] Sugiyama, Y., Xue, Y.X. and Mano, T. (1995) Transient Increase in Human Muscle Sympathetic Nerve Activity during Manual Acupuncture. The Japanese Journal of Physiology, 45, 337-345. http://dx.doi.org/10.2170/jiphysiol.45.337

[16] Hinder, R.A. and Kelly, K.A. (1977) Human Gastric Pacesetter Potential. Site of Origin, Spread, and Response to Gastric Transection and Proximal Gastric Vagotomy. The American Journal of Surgery, 133, 29-33. http://dx.doi.org/10.1016/0002-9610(77)90187-8

[17] Uchida, S., Kagitani, F. and Hotta, H. (2010) Neural Mechanisms of Reflex Inhibition of Heart Rate Elicited by Acupuncture-Like Stimulation in Anesthetized Rats. Autonomic Neuroscience, 157, 18-23.

[18] Kawakita, K. and Gotoh, K. (1996) Role of Polymodal Receptors in the Acupuncture-Mediated Endogenous Pain Inhibitory Systems. Progress in Brain Research, 113, 507-523. http://dx.doi.org/10.1016/S0079-6123(08)61105-X

[19] Kawakita, K., Shinbara, H., Imai, K., Fukuda, F., Yano, T. and Kuriyama, K. (2006) How Do Acupuncture and Moxibustion Act? Focusing on the Progress in Japanese Acupuncture Research. Journal of Pharmaceutical Sciences, 100, 443-459. http://dx.doi.org/10.1254/jphs.CRJ06004X

[20] Lin, X., Liang, J., Ren, J., Mu, F., Zhang, M. and Chen, J.D. (1997) Electrical Stimulation of Acupuncture Points Enhances Gastric Myoelectrical Activity in Humans. The American Journal of Gastroenterology, 92, 1527-1530.

[21] Chen, R. and Kang, M. (1998) Observation on Frequency Spectrum of Electrogastrogram (EGG) in Acupuncture Treatment of Functional Dyspepsia. Journal of Traditional Chinese Medicine, 18, 184-187. 